\title{
Overexpression of acetyl-CoA carboxylase in Aspergillus terreus to increase lovastatin production
}

\begin{abstract}
The present work describes the application of homologous recombination techniques in a wild-type Aspergillus terreus (ATCC 20542) strain to increase the flow of precursors towards the lovastatin biosynthesis pathway. A new strain was generated to overexpress acetyl-CoA carboxylase (ACCase) by replacing the native ACCase promoter with a strong constitutive PadhA promoter from Aspergillus nidulans. Glycerol and a mixture of lactose and glycerol were used independently as the carbon feedstock to determine the degree of response by the A. terreus strains towards the production of acetyl-CoA, and malonyl-CoA. The new strain increased the levels of malonyl-CoA and acetyl-CoA by $240 \%$ and $14 \%$, respectively, compared to the wild-type strain. As a result, lovastatin production was increased by $40 \%$ and (+)-geodin was decreased by $31 \%$ using the new strain. This study shows for the first time that the metabolism of Aspergillus terreus can be manipulated to attain higher levels of precursors and valuable secondary metabolites.
\end{abstract}

Keyword: Acetyl-CoA carboxylase; Aspergillus terreus; Homologous recombination; Lovastatin; Overexpression 